\title{
Molecular dynamics computer simulation of water flows in nanochannels ${ }^{\S}$
}

\author{
A. KUCABA-PIETAL ${ }^{1 *}$, Z. WALENTA ${ }^{2}$, and Z. PERADZYŃSKI ${ }^{3}$ \\ ${ }^{1}$ Department of Fluid Mechanics and Aerodynamics, Rzeszów University of Technology, \\ 8 Powstańców Warszawy Ave., 35-959 Rzeszów, Poland \\ 2 Department of Mechanics and Physics of Fluids, IPPT PAN, 21 Świętokrzyska St., 00-049 Warszawa, Poland \\ ${ }^{3}$ Institute of Applied Mathematics and Mechanics, Warsaw University, 2 Banacha St., 02-097 Warszawa, Poland
}

\begin{abstract}
The work presents the results of the simulations of water flows through narrow channels (Poiseuille flows) performed using the molecular dynamics method, for two different channel widths (equal to 5 and 10 diameters of the water molecule) and for two different materials of the channel walls (copper and quartz).

In the simulations, physical properties of the materials and their electrostatic interactions were considered. The obtained results are compared with the analytical solutions for a micropolar fluid flow taking account of the experimentally obtained rheological constants of water.
\end{abstract}

Key words: nanoflows, micropolar fluid, molecular dynamics simulation, nanochannels.

\section{Introduction}

Although the continuous medium model, generally applied to describe fluid flows, is enormously useful, it does not take into account all the factors that occur in the molecular scale. It omits, for example: rotation and deformation of molecules, electrokinetic effects, which can be seen at the wall-fluid boundary, temperature jump, and the capillarity effect. They all manifest themselves when the observed phenomenon approaches the molecular scale and cause discrepancies between the results obtained experimentally and those based on the continuous medium model.

The problem has become particularly conspicuous in recent years, when, due to the progress in micro and nanotechnology, micro and nanodevices have begun to be built on a large scale with flow elements of a diameter smaller than one micrometer (sometimes of the order of a few nanometers). Microchannels belong to the basic elements of such devices. The determination of flow quantities is essential for the correct design and effective operation of both micro and biomedical devices.

The experimental results, e.g. [1, 2], explicitly indicate that microscale flows are essentially different from flows in macroscale and that Navier-Stokes description is not capable of explaining the observed effects. During the flow through very narrow channels, the calculated hydrodynamic quantities for a fluid as a continous medium (from classical solutions of Navier-Stokes equations) differ significantly from those obtained experimentally, and the difference increases with decrease of the channel diameter. It seems that the flow of the fluid in micro and nanochannels should be described at the molecular level. At the same time, experimental data agree well with those obtained on the basis of the micropolar fluid theory proposed by Eringen in 1966 [3], which is grounded on the assumption of a continuous medium but additionally takes into account microrotation of the molecules (microrotation spinning motion of molecules, independent of the rotation of flow velocity field).

The experiments and theoretical estimations indicate, that for real flows through narrow channels the importance of the micropolar effects grows when the channel width decreases down to the values comparable with the dimensions of the particles of the fluid. However, under such conditions the assumption of continuous medium, essential for the theory of micropolar fluids, does not seem to be justified. The problem of validity of the micropolar fluid model for the flows through narrow channels is yet to be solved.

The limit of the applicability of the micropolar fluid theory for microchannel flow can be predicted with reasonable accuracy on the base of papers [4-7]. The papers present the results of computer simulations of Poiseuille flow, obtained by molecular dynamics methods, and compare them with analytical solutions of the micropolar fluid flow equations (obtained on the assumption of a continuous medium). The comparison shows, that analytical solutions for velocity of Poiseuille flow at channel widths not smaller than 10 diameters of the molecule, are in reasonable agreement with the results of MD simulation. In addition, the simulation demonstrates that the molecules of the flowing medium are subject to microrotation and this microrotation is non-zero at the walls. However, it must be noticed that the mentioned results were obtained for very simple molecular models that may represent such fluids as $\mathrm{Cl}_{2}$ and $\mathrm{P}_{6}$, and for highly idealised models of the walls. The simulations did not take into account any aspects of

\footnotetext{
$\S$ Presented at $1^{\text {st }}$ National Conference of Nano and Micro Mechanics (KKNM08), Krasiczyn, July 2008.

*e-mail: anpieta@prz.rzeszow.pl
} 
a possible influence of the electric charge of the fluid on flow characteristics. Since flows in microdevices are most often those of water, whose molecule has electric dipole moment, or of the aqueous solutions of various substances, the question can be asked, whether for water the applicability limit of micropolar fluid theory is the same as for the flows of fluids, whose molecules do not have such electric dipole moment (e.g. $\mathrm{Cl}_{2}, \mathrm{P}_{6}$ ). Also - what is the effect of the material of the bounding walls on the flow?

Most microchannels are made of quartz, copper or silicones [8-12]. The important question is what is the influence of the electric charge of the walls on the flow? - such a situation does occur in quartz channels and does not in copper ones. Apart from that it is important to know whether the values of material constants for liquid water, based on the data from the simulation, are equal to the values arrived at experimentally? The values of rheological constants in the analytical equations were predicted in $[4,6]$ on the basis of the data from the computer simulation.

The goal of the research reported in the paper was to answer the questions formulated above. In the following we present the results of calculations for water flows through nanochannels with the use of the molecular dynamics method. Planar Poiseuille flow through slits with copper and quartz walls were simulated. All the parameters concerning the molecular structure of the material were taken from the literature [13, 14]. Electric interactions, wherever present, were taken into account. The simulations were carried out for channels of two different widths: 5 and 10 diameters of water molecules.

The obtained results were compared with the analytical solutions of Eringen's equations for micropolar fluid flow. The values of material constants for water obtained from the results of the MD simulation were compared to those experimentally determined. Reasonable agreement was obtained for the flows in the channels of widths equal to 10 diameters of water molecules. For narrower channels the agreement was poor. Velocity slip on the walls could be observed. The calculation showed that the channel width and the kind of material of the wall affect the slip length of water flow.

Moreover the results suggest the application of micropolar fluid of non-zero microrotation on the walls in analytical solutions.

\section{Plane Poiseuille flow of a micropolar fluid}

Poiseuille flow is one of a few problems for which the solution of the micropolar fluid flow equations may be found in analytical form. For the plane channel of width $h$, in the Cartesian coordinates $(x, y, z)$ the solutions of equation can be written as [15]

$$
\begin{gathered}
\frac{V_{x}}{V_{0}}=1-\bar{z}^{2}+\frac{2 \delta_{0} \xi}{\left(2+\delta_{0} \xi\right)}\left(\frac{\cosh (k \bar{z})-\cosh (k)}{k \sinh (k)}\right), \\
\frac{\omega_{y} h}{V_{0}}=\left(\bar{z}-\frac{\xi\left(2+\delta_{0}\right)}{2+\delta_{0} \xi} \frac{\sinh (k \bar{z})}{\sinh (k)}\right),
\end{gathered}
$$

where $\boldsymbol{V}=\left(V_{x}, 0,0\right)$ - velocity, $\boldsymbol{\omega}=\left(0, \omega_{y}, 0\right)$ - microrotation, $\bar{z}=z / h$. Parameters $k$ and $\delta=\delta_{0} \xi$ can be found experimentally for particular fluid [16]. They are related to the rheological constants (i.e.viscosity coefficients) of micropolar fluid and fluid-channel interactions. For water flowing through quartz microchannels the values of these parameters are: $k=70.3 \cdot 10^{-7} \mathrm{~m}^{-1}, \delta=1.64$ [16].

\section{Molecular Dynamics simulation of plane Poiseuille flow}

3.1. Molecular models of materials. In Cartesian coordinates water was flowing in the in the $x$ - direction between two plane walls perpendicular to the $z$-axis. The flow was induced by an application of the constant force $F_{x}$ to the mass centre of every water molecule. The calculation programme based on the "MOLDY" programme by K. Refson from Oxford University [17] was written to simulate the flow. The molecular parameters of the materials were taken from the literature $[13,14,18]$. The geometry of the molecular models of water, quartz and copper are shown in Figs. 1 and 2. According to the assumed molecular models, the interactions between molecules of water, as well as water and the wall molecules were described with the Lennard - Jones potential [14]. The molecular interactions of atoms in quartz crystal were described with the Buckingham potential [13] and the interactions between atoms of copper with the Generic potential. a)

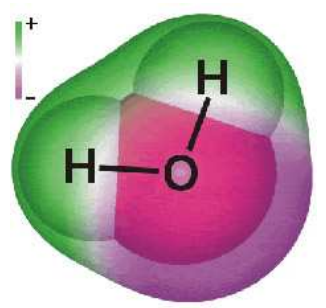

b)

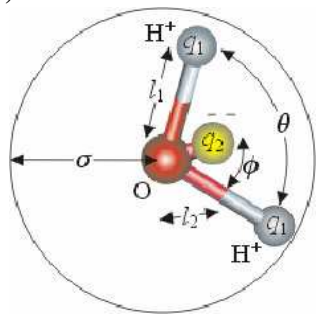

Fig. 1. Water molecule: a) the structure of a water molecule illustrating the density and distribution of electric charge.b) a schematic representation of the TIP4P molecular model geometry of a water molecule for the simulation: $l_{1}=0.9572 \cdot 10^{-10} \mathrm{~m}, l_{2}=0.15 \cdot 10^{-10} \mathrm{~m}$, $q_{1}=+0.5200, q_{2}=-1.0400, \theta=104.52^{\circ}, \phi=52.26^{\circ}$. The value of the charge is related to the value of elementary charge, $1.60^{*} 10^{-19} \mathrm{C}$, after Ref. 18 a)

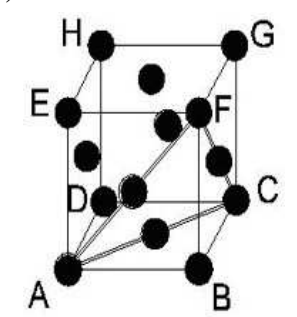

b)

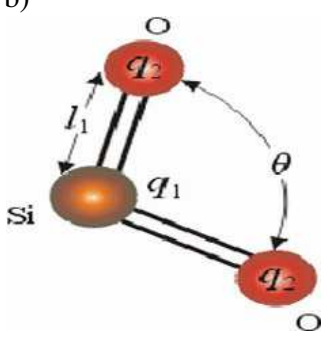

Fig. 2. Schematic representation of the geometry of molecular material models: a) elementary copper crystal after Ref. 14, b) geometry of the molecular model of a single quartz molecule; $l_{1}=$ $1.60 \cdot 10^{-10} \mathrm{~m}, \theta=109.28^{\circ}, q_{2}=+2.4, q_{1}=-1.2$ after Ref. 18 . The value of charge is related to the value of elementary charge, $1.60^{*} 10^{-19} \mathrm{C}$, after Ref. 13

The total potential energy $U$ of the simulated system consists of the energy $U k$, determined by the energetic potential 


\section{Molecular dynamics computer simulation of water flows in nanochannels}

describing short range interactions and resulting from the assumed molecular models of water, quartz and copper, as well as of the energy $U e$, which comes from the system of electric charges distributed on the water and quartz molecules and which is determined by the electrostatic interaction potential describing long-range Coulombian interaction. The so called Ewald sums [15] were applied to describe the energy $U e$ originating from the charges distributed on the molecules.

3.2. The structure of the unit simulation cell. In the performed simulation, MD unit cell was in the form of a rectangular prism and contained water molecules bounded by walls consisting of another kind of molecules/atoms. The number of water molecules in the MD cell with quartz walls ranged from 280 for the narrower channel to 560 for the wider channel. Each quartz wall consisted of $5^{*} 5^{*} 2$ single quartz crystal cells; each quartz cell consisted of 3 quartz molecules. The oxygen atoms from quartz molecules in the walls were adjacent to the water layer. In the case of copper walls, each wall consisted of $14 * 8 * 3$ copper atoms. The narrower channel the MD unit cell contained 360 water molecules, whereas the wider channel contained 720 water molecules. The system was surrounded by periodic images of itself in each of the three Cartesian dimensions.

Table 1 summarises the dimensions of the elementary cells used for the simulation, the values of the width $H$ and those of the effective width of the flow slot $h$ (the unit is $1^{*} 10^{-10} \mathrm{~m}$ - angstrem); other notation as in Fig. 3.

Table 1

Dimensions of elementary cells used in simulations

\begin{tabular}{ccccccc}
\hline \hline$H$ & $h$ & $L x$ & $L y$ & $L z$ & $\begin{array}{c}\text { Kind of wall } \\
\text { materials }\end{array}$ & $\begin{array}{c}\text { Number of water } \\
\text { molecules } n\end{array}$ \\
\hline 34.6 & 31.5 & 24.52 & 21.23 & 43.63 & Quartz & 560 \\
\hline 18.9 & 15.75 & 24.52 & 21.23 & 28.89 & Quartz & 280 \\
\hline 34.6 & 31.5 & 25.27 & 27.88 & 38.22 & Copper & 720 \\
\hline 18.9 & 15.75 & 25.27 & 27.88 & 21.42 & Copper & 360 \\
\hline
\end{tabular}

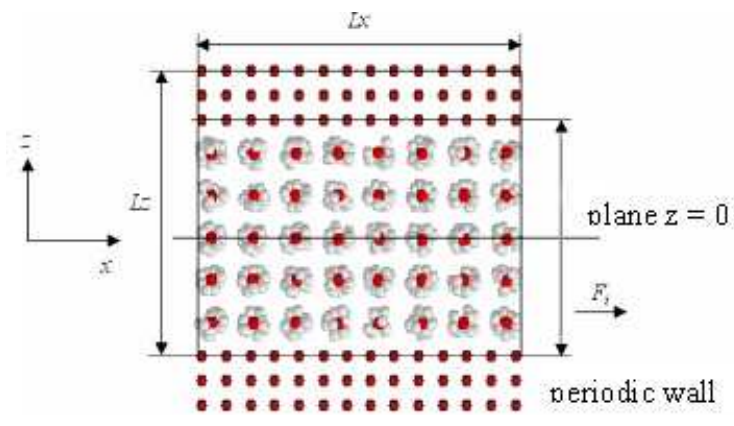

Fig. 3. Geometry of the cells used for the simulation

The definitions of the channel width $H$ and the effective channel width $h$ were introduced following the papers [4, 19]. The width $H$ was defined as a distance in the direction of the $O z$ axis between the planes going through the centres of atoms adjacent to the fluid. The effective channel width $h$ was defined as $h=H-\sigma$, where $\sigma$ is the value of the constant from the Lennard - Jones potential which describes the interaction between water and wall molecules. In accordance with the assumed model of water TIP4P [14], this value is equal to $\sigma=3.15^{*} 10^{-10} \mathrm{~m}$ ( $\sigma$ defines the linear dimension of the water molecule Fig. 1b.) In the MD simulations, physical quantities are usually given as reduced units, i.e. related to the quantities $\mathrm{m}, \sigma, \varepsilon$, where $\mathrm{m}$ is the mass of the molecule, $\sigma$ the characteristic length, and $\varepsilon$ the characteristic energy as defined for Lennard - Jones potential. Their values are marked with an asterisk [1]. Dimensionless units provide several computational benefits, such as numerical values close to unity and simplifying the equations of motion. However, the most useful benefit of using dimensionless units is that a single model represented in dimensionless units can be scaled for different problems.

The characteristic units for time and temperature are respectively: $\tau *=\sigma^{-1}(m / \varepsilon)^{-1 / 2} t, T^{*}=\left(\varepsilon / k_{B}\right)$, where: $k_{B}$ is Boltzmann constant, the density $n^{*}$ is defined by the formula: $n^{*}=N \sigma^{3} / v(v-$ the cell volume $)$ and the force: $F^{*}=F \sigma / \varepsilon$. For the molecular model of water TIP4P assumed for the calculation, the reference quantities have the following values: the mass of the water molecule $m=18$ a.m.u, (atomic mass unit) $\sigma=3.15 \cdot 10^{-10} \mathrm{~m}, \varepsilon=0.6480 \mathrm{~kJ} / \mathrm{mol}$. After substitution we get $\tau_{0}=m^{1 / 2} \sigma \varepsilon^{-1 / 2}=2 \cdot 10^{-13} \mathrm{~s}$, $T_{0}=k_{B} / \varepsilon=82 \mathrm{~K}$. Consequently, in the reduced units the parameters of the simulation for water are: temperature $T^{*}=3.6(300 \mathrm{~K}), n^{*}=0.997$. The time step for integration of the equations of motion $-\tau^{*}=0.002$. For quartz and copper, the values of the density are $n^{*}=1.13$ and $n^{*}=1.625$ respectively. The simulations were carried out for the force $F^{*}=2.5[15]$.

\section{Results}

The hydrodynamic parameters of the flow (distributions of velocity, microrotation and density) were obtained by averaging the data from 200000 time steps with the bin method [4, 15, 19]. The data were binned in one hundred layers in both, the wider and the narrower channels.

4.1. Density. The density $n^{*}$ (sometimes called as number density) and "physical density" $\rho\left[\mathrm{kg} / \mathrm{m}^{3}\right]$ are connected by formula: $\rho=n^{*}$ multiplied by (molecule mass in a.m.u).

When $\rho$ varies, then $n^{*}$ varies in the same way, so $n^{*}$ can be used to present the change of the flowing medium density across the channel too.

Presenting this number density variation $n^{*}$ we follow the line of the majority of papers devoted to MD simulations of nanoflows in nanochannels.

In Fig. 4. the obtained distributions of water density $n^{*}$ in the quartz and copper channels are presented.

The results show that the distribution of the water number density $n^{*}$ in the channel is non-uniform and symmetric. The greatest oscillations occur at the walls. Widening of the channels, both of copper and quartz, brings about enlargement of the area where the density approaches the value $n^{*}=0.997$. This area ranges from $20 \%$ in the narrower channel to $80 \%$ in the wider one. 
a)

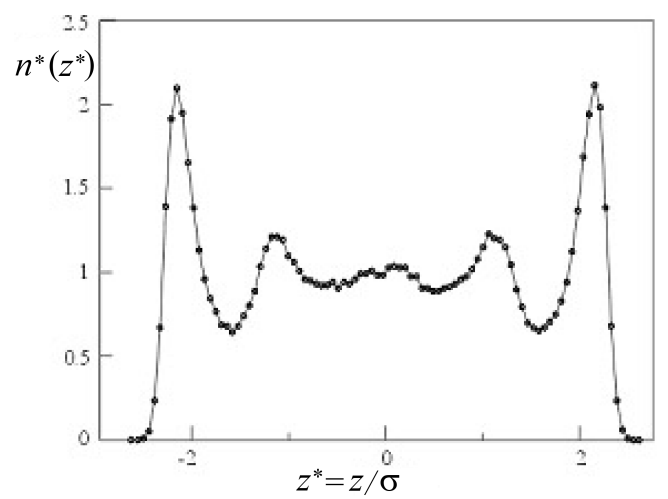

b)

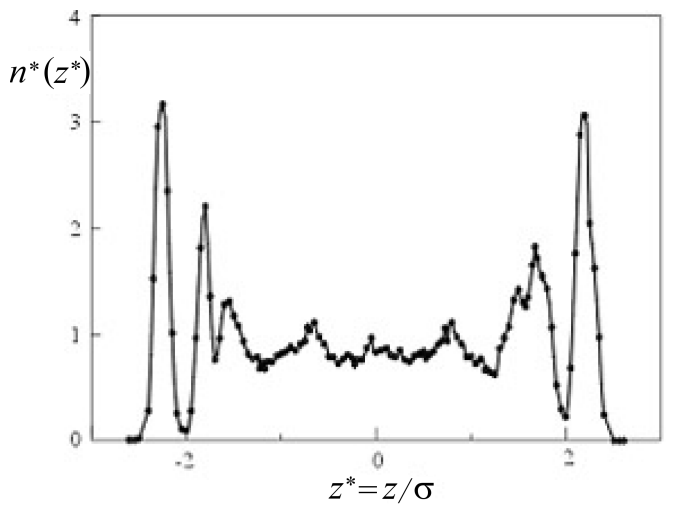

c)

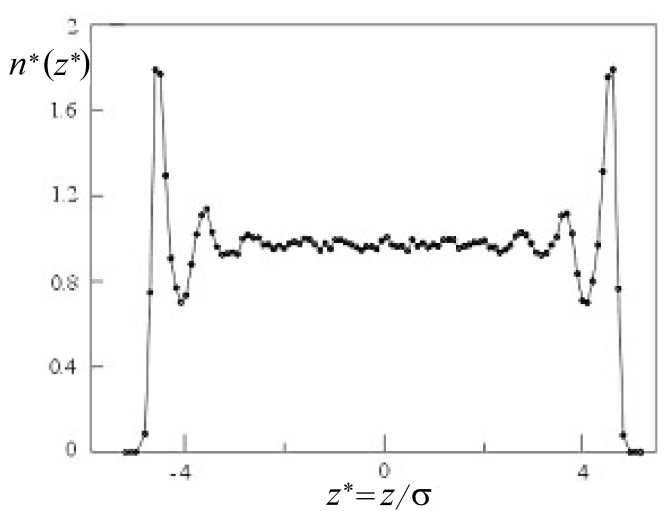

d)

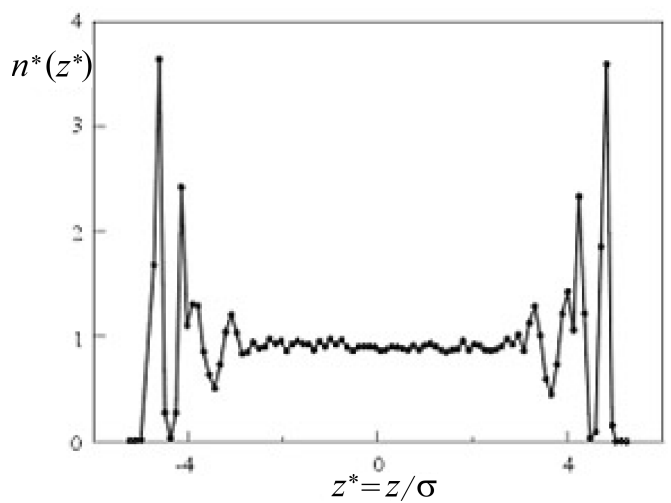

Fig. 4. Distributions of water density $n^{*}$ in the narrower (a), (b) and wider channels (c), (d) in copper: (a), (c) and quartz channels: (b), (d) after Ref. 15
A comparison of the obtained profiles of the water density, $n^{*}$, in the quartz and copper channels shows some differences in their shapes. In the simulation of the flow in the quartz channels (Fig. 4c, d), the profile of $n^{*}$ oscillates more in the midsection, the second peak has a characteristic kink which is not observed for water flows in the copper channels (Fig. $4 \mathrm{a}, \mathrm{c})$. The maximum value of the peaks of the water density profile $n^{*}$ is greater than that for flows through the copper channels.

It seems that the reason for the above mentioned differences is in the material properties of the walls. The walls differ in the density and moreover there are electric charges on the quartz molecules and none on the copper atoms. The ratio of the water density to the wall density for quartz walls equals $1000 / 2650=0.377$, and in the case of the copper ones it is $1000 / 890=0.16$ [18].

The obtained results concerning the shape differences in the density distribution $n^{*}$ partly coincide with those obtained by Cieplak, $[19,20]$ They used the MD method to investigate Poiseuille flows in channels of constant wall density $\left(n s^{*}=0.85\right)$ for various fluid densities (the value of $n p^{*}$ ranged from 0.004 to 0.773 ). The results indicate that the value of $n p^{*}$ determines the number of peaks on the profile as well as their shape (Fig. 1 in [19]). The greater the $n p^{*}$ value (and also the value of the ratio $n p^{*} / n s^{*}$ ), the more peaks appear. They assume step-like shapes (the counterpart of "peak kink" in Fig. 4b, d).

4.2. Velocity. Figure 5 presents the velocity profiles $V_{x}^{*}=$ $V_{x} \tau_{0} / \sigma$ obtained from the simulation of the water flow induced by the applied external field $F_{x}^{*}=2.5$ together with the approximating curves obtained from the analytical solution [15].

In the case of the narrower channel (Fig. 5a, c) the velocity profile is markedly different from the parabolic shape. It has three local maxima. Profiles of a similar shape were obtained in the MD simulation of Poiseuille flow of a fluid composed, of monoatomic molecules through channels of effective width $h=3$ and $h=4$ [18].

As in Travis' work [21], the curve:

$$
V_{T}\left(z^{*}\right)=a_{0}+a_{1} z^{* 2}+\sum_{n=2}^{n_{\max }} a_{n} \cos \left[2 \pi \frac{(n-1) z^{*}}{h^{*}}\right]
$$

was taken for the approximation obtained water nanoflow MD results for the narrower channel.

For the wider and narrower channel the results of the MD simulations additionally were compared with the analytical curve (1), using the values of the constants, obtained experimentally for water.

Applying the method of the least nonlinear squares (Genfit procedure from the MathCAD programme), the values of the constants $k 1=k \cdot h, D 1=\frac{2 \delta}{2+\delta}$ and $V_{0}$ in the analytical solution (1) were determined using the data from the MD simulation. 
a)

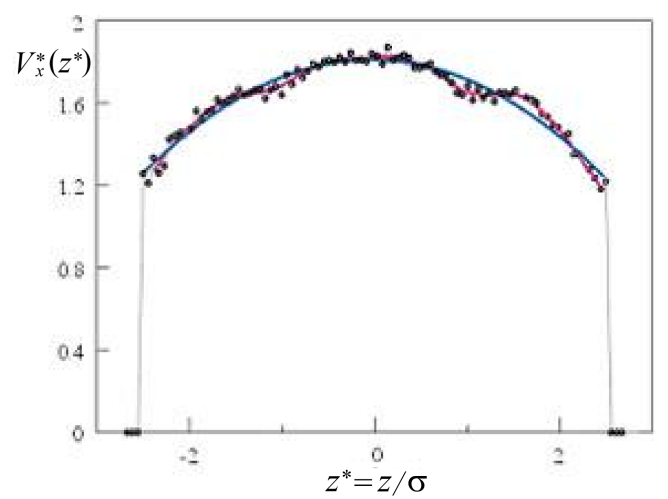

b)

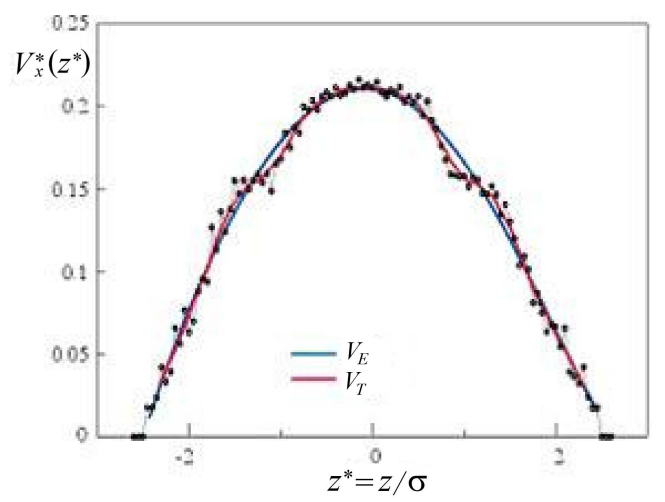

c)

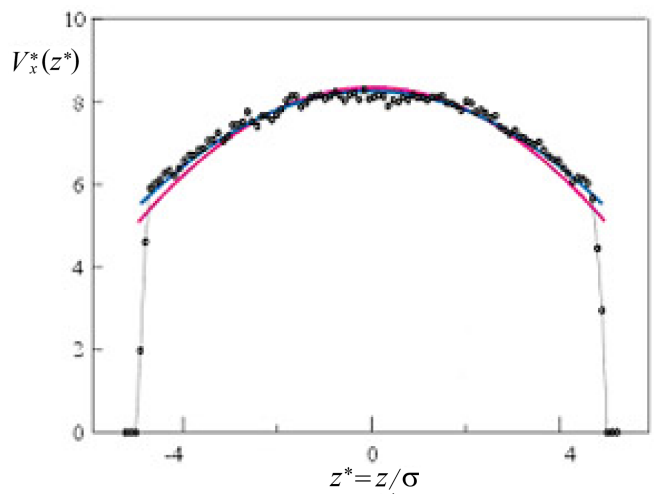

d)

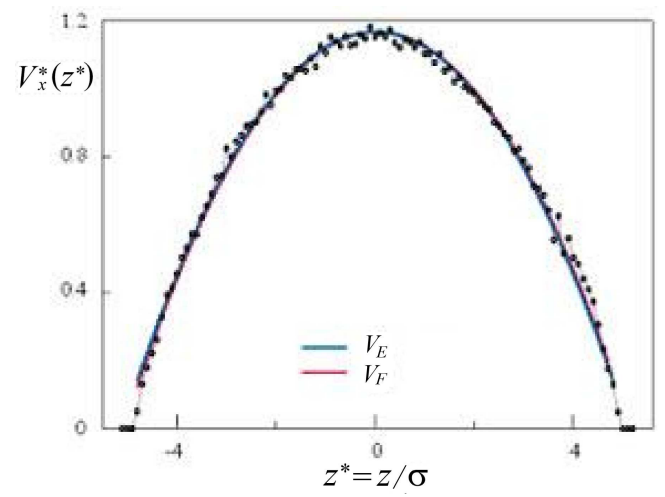

Fig. 5. Water velocity distributions at $F x^{*}=2.5$ in copper (a), (c) and quartz (b), (d) channels: in the narrower (a), (b) and the wider ones (c), (d) with approximating curves obtained from: $V_{E}$ - analytical solution (1), $V_{T}$ - Travis' curve. Points o denote the values from the simulation
The obtained numerical values were: $k 1=0.33 \pm 0.09$, $D 1=0.1 \pm 0.07, V 0=0.46 \pm 0.09$.

The experimentally obtained data permit exact evaluation of $k 1=k \cdot h$ only. The value is equal to 0.05 for the narrower channel and 0.105 for the wider.

The experimentally obtained value $\delta=1.45$ gives $D 1=$ 0.8 for wider and for narrower channel too.

For the wider channel, the value of $k 1$ from approximation is about three times larger than that from experiment, for the narrower channel the value of $k 1$ from approximation is nearly ten times larger than that from experiment.

At the moment we are unable to answer this question what caused the discrepancies. To give the proper answer, an additional study would be required. It should be only underline that the experimentally obtained value were predicted for water flowing through the microchannel with quartz walls [16].

Fluid flow in confined geometries can be significantly affected by slip at the liquid/solid interface. Molecular dynamics simulations provide a powerful tool for studying the effect of these parameters on boundary conditions at the molecular level. The measure of slip is the so-called "slip length", which is defined as the distance between the wall and the point where the extrapolated tangential velocity component vanishes.

In both wider and narrower channel the velocity slip on the walls can be observed.

Results show that the slip length decreases with the increase of the channel width.

As expected, maximum velocity values in the two channels are different too; in the wider one the velocity is 4.5 times larger.

A comparison of the velocity distributions in the copper and quartz channels shows that in the latter the water velocity slip on the walls is smaller.

The maximum value of the water velocity $V x^{*}$ is also smaller than that for the flow through copper channels.

The reasons of the above velocity differences, as well as density differences, can be attributed to the material properties of the walls (different for various values of the ratio of water density to wall density and depending also on the presence of electric charges in quartz wall on the flow - water molecules possess electric dipole moment). The same differences in the shapes of velocity profiles were observed by Cieplak during the MD simulations of dense gas flows through narrow channels with attractive and repulsive [20,21] walls. A comparison of the obtained results for water with those, mentioned in [20, 21] suggests that the copper wall repulses water molecules, whereas the quartz wall attracts them.

On the other hand it seems, that the effect of various wall densities on the water flow velocity cannot be totally excluded, as the results obtained by Cieplak [20] show that the magnitude of slip depends on the properties of the molecular model of the walls and on the fluid density. The results of Thompson's research [12] on the MD Couette flows of fluids of various densities also show that one can have different slip values on the wall at different densities. 
a)

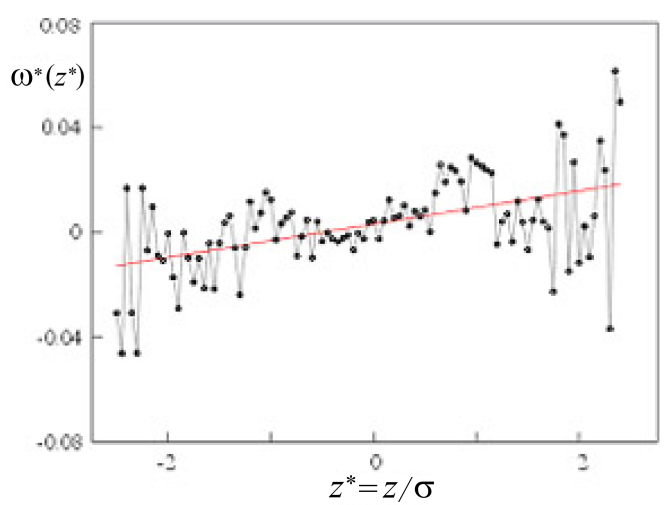

b)

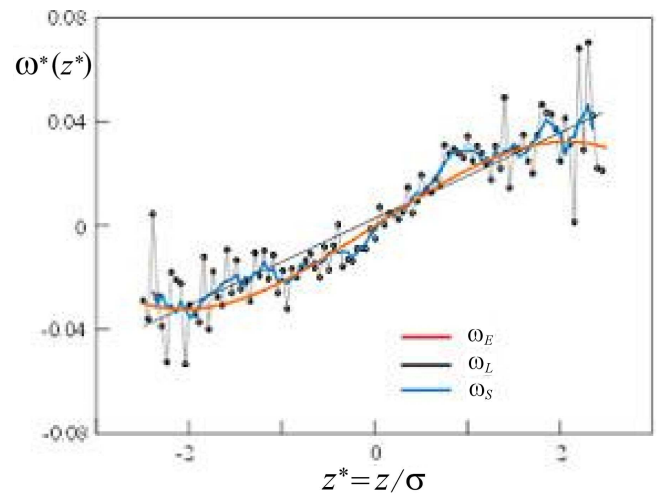

c)

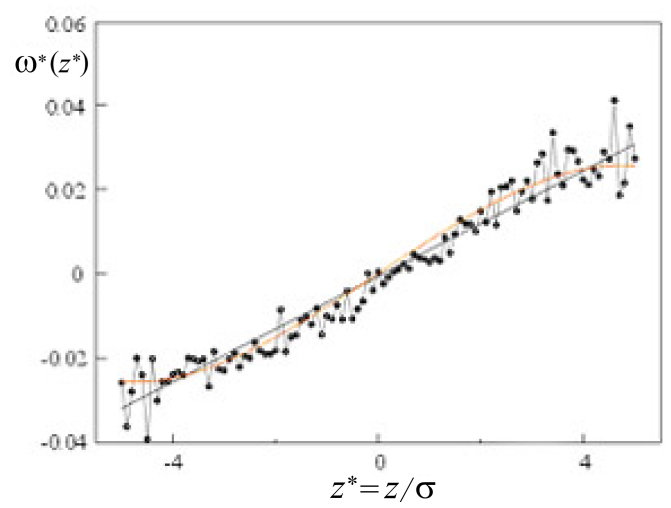

d)

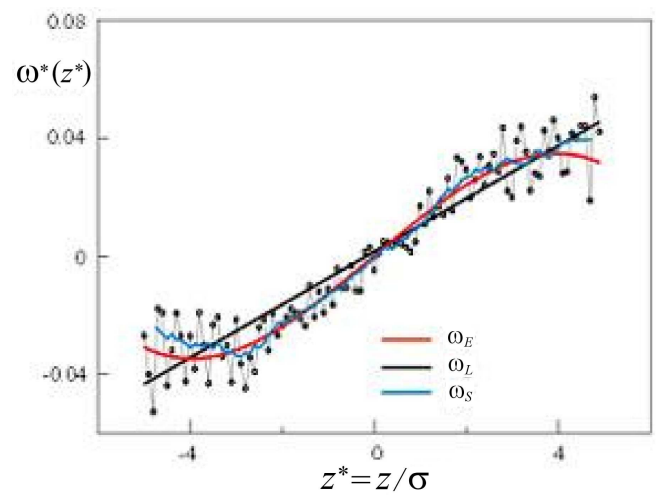

Fig. 6. Distributions of water microrotation at $F x^{*}=2.5$ in the copper (a), (c) and quartz channels (b), (d): in the narrower (a), (b) and wider ones (c), (d) with approximating curves obtained from: $\omega_{E}$ - analytical solution (2), $w_{l}$ - linear function. Points o denote the values from the simulation
4.3. Microrotation. Microrotation distributions $\omega^{*}=\omega \tau_{0}$ in the quartz and copper channels were given in Fig. 6.

It can be noticed, that:

- In the narrower channel, at the distances of the order of $\sigma$ from the centerline, good agreement with the approximating curve has been obtained; further on there appear strong oscillations, increasing when approaching the wall,

- There is non-zero microrotation at the walls,

- In the wider channel, the microrotation velocity is in reasonable agreement with the profile obtained from the micropolar fluid theory, except for some peculiarities at the walls which are probably due to molecular packing.

\section{Conclusions}

The novelty of the results presented in the paper is, that they were obtained based on the real physical parameters of the media: water, copper and quartz. To our knowledge it is the first time that the real electrostatic forces acting between the wall material (quartz) and the flowing medium (water) were taken into account in MD simulation of nanoflows.

At the first step we performed the molecular dynamics simulations of water flows under gravity field down rectangular channels.

Next we compared Poiseuille micropolar theoretical predictions for flow velocity and microrotation with the above mentioned results of molecular dynamics simulations. In micropolar equations describing the flow the real experimental rheological parameters were used.

- The obtained results show influence of the electric charges of channel walls and flowing water molecules on flow characteristics. The MD results show, that for channel walls made of copper the water velocity slip effects are stronger than those for walls made of quartz. Water and quartz molecules do have some electric charge, copper does not. It results in arising long range, attractive electrostatic forces between quartz molecules and water molecules. As a result, quartz is attractive for water and copper is not.

- The results show that for channel widths equal to 10 diameters of the molecule the results of the micropolar theory are in a reasonable qualitative agreement with molecular dynamics simulation. In particular, the microrotation velocity (missing in the classical Navier-Stokes theory) and the translational streaming velocity profiles agree well with those from MD simulation. The analysis shows that the geometric size of flow fields plays a crucial role in the useful applicability micropolar fluid theory to modelling nanoflows [23].

- In all cases under consideration some slip velocity and nonzero microrotation at the walls could be observed. The slip effects are stronger for smaller channel widths and for walls made of copper. They seem to be influenced by interaction of water molecules - which have electric dipole moment with the charges of the walls. Quartz does have some electric charge, copper does not. For water molecules the quartz 


\section{Molecular dynamics computer simulation of water flows in nanochannels}

wall behaves as an attractive one, whereas the copper wall (without charge) as a repulsive one.

- The obtained results are consistent with earlier research. The effect of attractive and repulsive walls on the slip velocity was observed in Poisseuille flow simulations of dense gas flows in narrow channels [20,21]. The present results, however, are the first obtained for flows of fluid whose molecules possess electric dipole moment.

The problem of interaction between fluid and solid surfaces has received increased attention over the past ten years as engineering applications reached the micrometer and nanometer scale. For the past century the non-slip boundary condition was considered to be appropriate for macroscopic flows. Our results, as well as experimental and simulation results of other authors show that the slip velocity in micro and nanoflow exists and strongly depends on wall - fluid interaction.

\section{REFERENCES}

[1] M. Gad-El-Hak, "The fluid mechanics of microdevices", $J$. Fluids Engng. 121 (5), 1215-1233 (1999).

[2] K.V. Sharp, R.J. Adrian, J.G. Santiago. and J.I. Molho, "Liquid flows in microchannels", in Handbook of MEMS, ed.M. Gad-el-Hak, Virginia Commonwealth University, Richmond, USA, 2001.

[3] A.C. Eringen, "Theory of micropolar fluids", J. Math. Mech. 16 (1), 1-16, (1996).

[4] J. Delhommelle and D.J. Evans, "Poiseille flow of micropolar fluid”, Molecular Physics 100 (17), 2857-2865 (2002).

[5] D.C. Rapaport, "Shear-induced order and rotation in pipe flow of short-chain molecules", Europhysics Letters 26 (6), 401406 (1994)

[6] K.P. Travis and D.J. Evans, "Molecular spin in a fluid undergoing Poiseuille flow", Phys. Rev. E. 55 (2), 1566-1572 (1997).

[7] K.P. Travis, B.D. Todd, and D.J. Evans, "Poiseuille flow of molecular fluids", Physica A 204, 315-327 (1997).

[8] R.R. Reston and E.S. Kolesar, "Silicon-micromachined gas chromatography system, Part I. Design and fabrication", IEEE J. Microelectromech. Sys. 3-4, 134-142 (1994).
[9] H.A. Stone, A.D. Stroock, and A. Ajdart, "Engineering flows in small devices: micro-fluidics towards a lab-on-a-chip", Ann. Rev. Fluid Mechanics 36, 381-411 (2004).

[10] P. Tabeling, "Some basic problems of microfluidics", $14^{\text {th }}$ Australian Fluid Mech. Conference 10-14 Dec. 2001, (2001).

[11] P. Wilding, M.A. Shoffner, and L.J. Kircka, "Manipulation and flow of biological fluids in straight channels micromachined in silicon", Clin. Chem. 40, 43-47 (1994).

[12] P.A. Thompson and S.M. Trojan, "A general boundary condition for liquid flow at solid surfaces", Nature 389, 360-361 (1997).

[13] B.W.H. van Beest, G.J. Kramer, R.A. van Santen, "Force fields for silicas and aluminophosphates based on ab initio calculations", Phys. Rev. Lett. 64 (16), 1955-8 (1990).

[14] http://www.lsbu.ac.uk/water/

[15] A. Kucaba-Piętal, "Microflows modelling based on micropolar fluid theory", Oficyna Wydawnicza Politechniki Rzeszowskiej, Rzeszów 2004 (in Polish).

[16] P.Prokhorenko, N.P. Migoun, and M.Stadthaus, Theoretical principles of liquid penetrant testing, DVS Verlag, Berlin 1999.

[17] K. Refson, "Moldy: a portable molecular dynamics simulation program for serial and parallel computers", Computer Physics Communications 126 (3), 309-328 (2000).

[18] Metals Reference Book, 5 edition, Butterworth, London 1976.

[19] K.P. Travis, B.D. Todd, and D.J. Evans, "Departure from Navier-Stokes hydrodynamics in confined liquids", Phys. Rev. E 55 (4), 4288-4295 (1997).

[20] M. Cieplak, J. Koplik, and J.R. Banavar, "Applications of statistical mechanics in subcontinuum fluid dynamics", Physica A 274, 281-293 (1999).

[21] M. Cieplak, J. Koplik, and J.R. Banavar, "Molecular dynamics of flows in the Knudsen regime", Physica A 287, 153-160 (2000).

[22] K.P. Travis and K.E. Gubbins, "Poiseuille flow of LennardJones fluids in narrow slit pores", J. Chem. Phys. 112 (4), 1984-1996 (2000).

[23] A. Kucaba-Piętal, "Microchannels flow modelling with the micropolar fluid theory", Bull. Pol. Acad. Sci. Tech. 52 (3), 209-214 (2004). 